\title{
Frequency regulation by fuzzy and binary control in a hybrid islanded microgrid
}

\author{
Pamela MANJARRES, Om MALIK ( $\square)$
}



\begin{abstract}
Islanded microgrids must be self-sufficient in terms of frequency and voltage control due to their islanded operation. A control strategy for frequency regulation by combining the operation of a wind generator, a diesel generator, a battery energy storage system and a dump load in a microgrid is proposed in this paper. In the proposed strategy, the control task is partitioned into two subtasks: 1) choosing the appropriate element to be used for regulation, and 2) providing frequency regulation. A global controller chooses the element to operate. Then, the frequency regulation is provided by separate individual controllers. The proposed control strategy is tested on a microgrid with mixed types of generation and modeled on Simulink. By monitoring the power of individual elements and system frequency, it is shown that the proposed control strategy operates efficiently. The proposed strategy facilitates the integration of renewable energy sources and enhances frequency regulation.
\end{abstract}

Keywords Frequency regulation, Fuzzy control, Hybrid microgrid, Automatic operation

\section{Introduction}

Integration of renewable energy sources into power systems is a crucial step in reducing the environmental

CrossCheck date: 4 November 2014

Received: 17 February 2014 / Accepted: 13 November 2014 / Published online: 16 December 2014

(C) The Author(s) 2014. This article is published with open access at Springerlink.com

P. MANJARRES, O. MALIK, Department of Electrical and

Computer Engineering, University of Calgary, Calgary, AB, Canada

(凹) e-mail: maliko@ucalgary.ca impact of electric power generation. Renewable energy can substitute the usual fuel-based sources of energy resulting in environmentally friendly power systems. However, renewable energy sources, such as wind power, being intermittent can be unreliable. As a result, weather dependent sources cannot be used alone to efficiently supply a load.

Microgrids with a variety of power generation sources tackle the unreliability issue by combining renewable energy sources with controllable fuel-based generation. The fuel-based generation provides reliable power while the renewable energy reduces the environmental impact of generating electric power.

As these systems, often referred to as microgrids, may sometimes operate in an islanded mode, a control approach is required to maintain frequency and voltage regulation. Control of system frequency only is considered in this paper.

Frequency is dependent on the real power balance in the system. Hence, balancing mechanisms can be introduced to the system with the purpose of offsetting the intermittent power generation of a renewable energy.

Various energy sources have been used to provide frequency regulation in islanded hybrid microgrids. In [1] and [2], frequency is regulated through dump loads (DL), while a battery energy storage system (BESS) is used as the main frequency regulator in [3] and [4].

Operational requirements demand that the BESS operation be maintained between the State of Charge (SOC) limits and instantaneous power limits. Very few studies have considered the SOC when operating a BESS as a balancing mechanism in a hybrid power system, as it has been done in study [5]. This constraint is considered in this paper.

Using a single balancing mechanism for frequency regulation is a common topic in the literature. On the other hand, the combination of multiple balancing mechanism is a less visited topic. In this study, a power system that 
combines a diesel generator set (DG), a battery energy storage system (BESS) and a dump load (DL) as balancing mechanisms has been chosen. A wind generator (WG) is used as the renewable source of energy. The system schematic is shown in Fig. 1.

The use of multiple balancing mechanisms raises the need for coordination between the balancing mechanisms. Three balancing mechanisms are chosen in this study as were also used in [6] and [7]. A pre-set step signal can be used to initiate the change of operation mode. Although a pre-set signal is not used in the simulation studies in [6], it is shown by the same author in study [7] that this pre-set signal is required to commit the DG when preventing the discharge of the BESS. Thus, the strategy implemented in [6] and [7] is not completely automatic.

Other studies in the literature opted for calculating the system real power mismatch [5, 8-10]. The power mismatch estimates the difference between wind power production and load consumption. In [8] the power mismatch is used to generate an ON signal to commission the DG.

Studies in [9] and [10] balanced the power mismatch in order to extract maximum power from the wind turbine. However, the power mismatch calculation is again required to decide whether the DL is committed. In study [5] the power mismatch is used as an input of a fuzzy inference engine.

A control strategy that needs neither the load consumption nor the wind power production to operate the system is proposed in this paper. The coordination of balancing mechanisms is achieved by means of a inference engine that mimics a human operator decision. The proposed strategy divides the regulation task in two parts: 1) selecting a balancing mechanism and 2) providing frequency regulation.

A global controller has been designed using an IFTHEN inference engine that allows the coordination of balancing mechanisms without power mismatch calculation. Then, individual fuzzy controllers operate each balancing mechanism. High-order mathematical models are used to test the proposed control strategy.

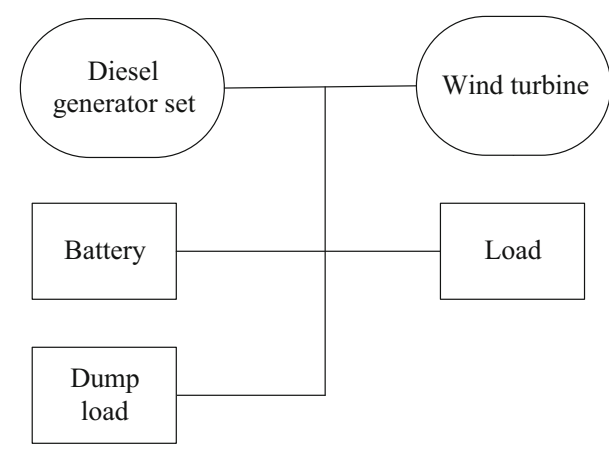

Fig. 1 Hybrid power system schematic
To enhance the frequency regulation, a fuzzy control is proposed to prepare the DG for commissioning. In previous studies, the diesel engine was operated only in two states: 1) rest, and 2) nominal speed. That approach results in a large frequency deviation as seen in [6]. Here, an engine controller has been designed to regulate the diesel engine speed according to the system needs.

The paper is organized as follows. The hybrid power system structure and mathematical model are discussed in Sect. 2. The hybrid power system operational guidelines are briefly described in Sect. 3. The overall control strategy is detailed in Sect. 4 and the global controller that allows the automatic operation of the system is described in Sect. 5. Details of the frequency and operational controllers are given in Sect. 6 and simulation results are given in Sect. 7. Finally, the conclusions and findings of this study are given in Sect. 8.

\section{Microgrid model}

The microgrid under study comprises a DG rated 300 $\mathrm{kVA}$, a WG rated $275 \mathrm{kVA}$, a BESS and a DL. The BESS instantaneous absorption and supply are limited between 150 and $+150 \mathrm{~kW}$, respectively. The DL maximum consumption is $200 \mathrm{~kW}$. All system elements are connected at $480 V_{\text {rms. }}$.

High-order mathematical models have been used to represent the microgrid in Simulink. The microgrid elements were modeled separately and then combined to form the microgrid under study.

The DG model consists of a diesel engine, a synchronous machine and a clutch. The diesel engine model is represented by separate high-order transfer functions as in [11]. The engine parameters were extracted from [12].

The synchronous machine model and parameters can be found in the Simulink SimPowerSystems library and follows the model in [13]. The clutch modifies the torque transmitted to the synchronous machine $[6,8]$ and the diesel engine speed [8]. When locking the clutch, a maximum speed difference of $10^{-5}$ is acceptable. Similar to the studies in $[6,7]$, the diesel engine cranking dynamics are avoided. To avoid the unnecessary diesel engine cranking dynamics, the engine rest state is simulated at a speed of 0.3 p.u. Thus, although the engine is considered stopped, the simulated value would remain at 0.3 p.u. Since the cranking delay will affect the readiness of the DG to provide frequency regulation, a $0.5 \mathrm{~s}$ delay is added as cranking time to the DG model $[6,7]$. Thus, the diesel engine start will be delayed by $0.5 \mathrm{~s}$ when the speed increases from 0.3 p.u.

The WG model comprises a wind turbine and an asynchronous generator. The wind turbine model follows a 
cubic relationship between wind speed and the harnessed mechanical power. The wind turbine mathematical model can be found in [14]. According to the power rating, a wind turbine radius of $20 \mathrm{~m}$ was chosen [15]. The asynchronous generator was implemented using high-order mathematical models for both electrical and mechanical parts. The mathematical model can be found in the Simulink SimPowerSystems library and follows the model in [13]. The asynchronous generator has a voltage and power rating of $480 V_{\text {rms }}$ and $275 \mathrm{kVA}$, respectively.

The BESS mathematical model follows [16]. In order to comply with power and voltage system requirements, a capacity of $117 \mathrm{Ah}$ and a voltage of $1,000 V_{\mathrm{DC}}$ were chosen. A Nickel Metal Hydride (NiMH) type of battery is used due to its environmental-friendly aspect [17]. The parameters of a single cell $\mathrm{NiMH}$ battery were extracted from study [16].

In addition, the SOC calculation has been modified to account for the Peukert effect. Based on study [18], a Peukert coefficient of 1.024 was selected. The SOC calculation follows [19]. To control the BESS power output, a current injection control is used similar to studies [20] and [21].

The DL comprises 8 electrical resistors that are switched to obtain the required power consumption. The model was developed following study [22]. The DL resistors presented the values of $230.4,115.2,57.6,28.8,14.4,7.2,3.6$ and 1.8 $\Omega$. Ideal switches were used to connect the resistors comprising the DL. To control the DL switches, a look-up table has been implemented as in study [22].

Voltage regulation is provided by the DG. The DG synchronous generator uses its excitation system to maintain the system's voltage between standards, as seen in [6]. The excitation system was modeled according to the IEEE Recommended Practice [23] for a DC1A Type Excitation System. In addition, a capacitor bank is implemented at the WG terminals to offset the asynchronous generator reactive power consumption.

\section{Microgrid operational guidelines}

In this study, the microgrid is operated according to an environmentally friendly dispatch. The environmentally friendly dispatch reduces the use of fuel-based generation and maximizes the use of renewable energy.

BESS allows the system to increase the use of renewable energy by absorbing power during high wind conditions and using the stored power later, when wind power generation is low. In this study, the BESS can only be charged from renewable energy. Hence, the BESS functions as the system's primary balancing mechanism.

However, operational guidelines state that the BESS can only actuate as long as its SOC is between limits.
Following [24], the SOC limits have been established at $30 \%$ and $70 \%$ for empty and full, respectively.

In addition, the BESS instantaneous power output must also be restricted due to the battery's charge and discharge rate limits. As a result, the instantaneous supply and absorption has been limited between +150 and $-150 \mathrm{~kW}$, respectively.

If the BESS operational limits are surpassed, the DG or DL must replace the BESS as frequency regulators. The DG and DL are considered secondary balancing mechanisms.

\section{Overall control strategy}

The proposed control strategy divides the control task into two subtasks: 1) choosing the balancing mechanism to operate, 2) providing frequency regulation with the chosen balancing mechanism. Hence, the system's operation is managed by a layered control strategy.

The first layer consists of a global controller that chooses the balancing mechanism to operate according to the system conditions. When a balancing mechanism is chosen, the global controller outputs ON/OFF status, in the binary form of 1 and 0 , respectively. The running status is then given to the second control layer.

An ON status signal allows for the chosen frequency controller to be connected to the balancing mechanism; hence, the balancing mechanism is able to provide frequency regulation. An OFF status disconnects the frequency controller from the balancing mechanism. When OFF, the balancing mechanism remains at its last operational set point.

Four controllers are used in the second control layer to operate the balancing mechanisms. If the BESS or DL are committed to operation, the frequency controllers modify the BESS and DL power output.

If the SOC limits are reached, a BESS unload controller reduces the BESS power output to zero in order to prevent the SOC limits from being surpassed.

In contrast to the BESS and DL, the DG possesses a built-in frequency regulator. The speed governor of the diesel engine is able to provide frequency regulation by maintaining the diesel engine speed at nominal [25]. Hence, the design of a frequency controller for the DG is not required. However, in order to improve the system performance, an engine controller has been designed to operate the diesel engine according to the system needs.

Outputs of the second control layer are the operational set points of each balancing mechanism. The operational set points are then given to individual operational controllers. Once the new operating points are reached, the control task is completed. A graphical representation of the 


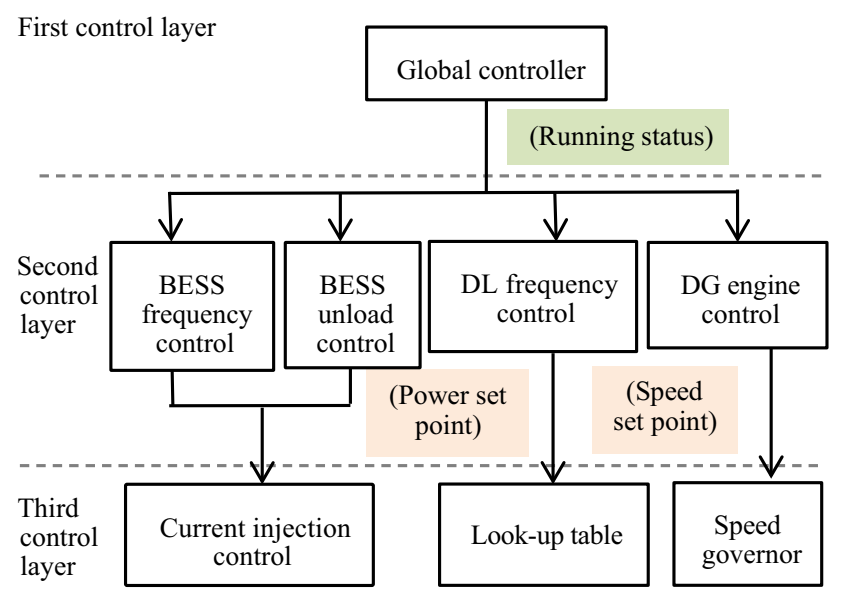

Fig. 2 Graphic representation of the hierarchical relationship among system controllers

hierarchical relationship between the control layers is depicted in Fig. 2.

\section{Global controller}

The global controller has been designed using knowledge based rules that allow the system to operate automatically. The rules are designed in an IF-THEN format, where the system conditions allow the IF clause to decide which balancing mechanism to operate.

The global controller monitors the BESS power output $\left(P_{\mathrm{BESS}}\right)$, the DG power output $\left(P_{\mathrm{DG}}\right)$, the DL power output $\left(P_{\mathrm{DL}}\right)$, the system frequency deviation $(\Delta f)$ and the SOC. As in fuzzy control, the crisp value of each input is transformed into a state. The input state is then used to choose the balancing mechanism to operate.

As the outputs of the global controller are binary, this controller functions as a binary control. Hence, the input states are also divided in a binary fashion. The control inputs and their states are summarized in Table 1.

As long as $P_{\mathrm{BESS}}$ is within Limits (i.e. $-150 \mathrm{~kW}$ $<P_{\mathrm{BESS}}<150 \mathrm{~kW}$ ) and $\mathrm{SOC}$ is Normal (i.e. $30.15 \%$ $<$ SOC $<69.85 \%$ ), the BESS is committed to frequency regulation, as shown in the control surface in Fig. 3. The control surface depicts the actuation of the global controller to a set of inputs. When the global controller output is ON, the surface presents a value of 1 . When the output is OFF, the surface presents a value of 0 .

The global controller considers the SOC is empty or full $0.15 \%$ before the actual SOC operating limits (i.e. $30 \%$ and $70 \%$ ). This is to allow for the BESS power output to be gradually reduced without surpassing the SOC operating limits.
Table 1 Global controller inputs

\begin{tabular}{ll}
\hline Input & States \\
\hline$P_{\text {BESS }}$ & Negative Limit $\left[P_{\mathrm{BESS}} \leq-150 \mathrm{~kW}\right]$ \\
& Negative Normal $\left[-150 \mathrm{~kW}<P_{\mathrm{BESS}} \leq-1 \mathrm{~kW}\right]$ \\
& Zero $\left[-1 \mathrm{~kW}<P_{\mathrm{BESS}}<+1 \mathrm{~kW}\right]$ \\
& Positive Normal $\left[+1 \mathrm{~kW} \leq P_{\mathrm{BESS}}<+150 \mathrm{~kW}\right]$ \\
& Positive Limit $\left[P_{\mathrm{BESS}} \geq+150 \mathrm{~kW}\right]$ \\
& Zero $\left[P_{\mathrm{DL}}<+1 \mathrm{~kW}\right]$ \\
$P_{\mathrm{DL}}$ & Positive $\left[P_{\mathrm{DL}} \geq+1 \mathrm{~kW}\right]$ \\
& Zero $\left[P_{\mathrm{DG}}<100 \mathrm{~W}\right]$ \\
$P_{\mathrm{DG}}$ & Positive $\left[P_{\mathrm{DG}} \geq 100 \mathrm{~W}\right]$ \\
& Negative $[\Delta f \leq-0.005 \mathrm{~Hz}]$ \\
$\Delta f$ & Zero $[-0.005 \mathrm{~Hz}<\Delta f<+0.005 \mathrm{~Hz}]$ \\
& Positive $[\Delta f \geq+0.005 \mathrm{~Hz}]$ \\
& Empty $[\mathrm{SOC} \leq 30.15 \%]$ \\
SOC & Normal $[30.15 \%<\mathrm{SOC}<69.85 \%]$ \\
& Full $[\mathrm{SOC} \geq 69.85 \%]$
\end{tabular}

If the BESS operational limits are reached, the global controller commits a secondary balancing mechanism to frequency regulation.

If the SOC limits are reached, the unload controller is used to reduces $P_{\mathrm{BESS}}$ to zero. Similarly, the commissioning of the secondary balancing mechanisms also depends on the SOC state. The DL is committed if SOC is Full. The DG is committed if SOC is Empty. It is noticed from Fig. 3 that the global controller allows the BESS to charge if SOC is Empty and to supply if SOC is Full.

If the BESS instantaneous power limits are surpassed, the global controller uses $\Delta f, P_{\mathrm{DG}}$ and $P_{\mathrm{DL}}$ as decision variables. The frequency deviation $(\Delta f)$ allows the global controller to recognize between power surplus and shortage. A negative $\Delta f$ suggests the system presents a real power shortage, while a positive $\Delta f$ suggests that a real power surplus exists.

For example, if $P_{\mathrm{BESS}}$ is at maximum absorption (i.e. $P_{\mathrm{BESS}} \leq-150 \mathrm{~kW}$ ) and a power surplus exists (i.e. $\Delta f>0)$, the DL is committed to frequency regulation, as seen in Fig. 4. Similarly, If $P_{\mathrm{BESS}}$ is at maximum supply (i.e. $P_{\mathrm{BESS}} \geq 150 \mathrm{~kW}$ ) and a power shortage exists (i.e. $\Delta f<0)$, the DG is committed to frequency regulation. Once a secondary balancing mechanism is committed, it will remain as the actuating balancing mechanism until its power output is reduced to zero.

\section{Frequency and operational controllers}

Four controllers have been designed to operate the balancing mechanisms according to the system needs. The following subsections detail the second layer controllers. 


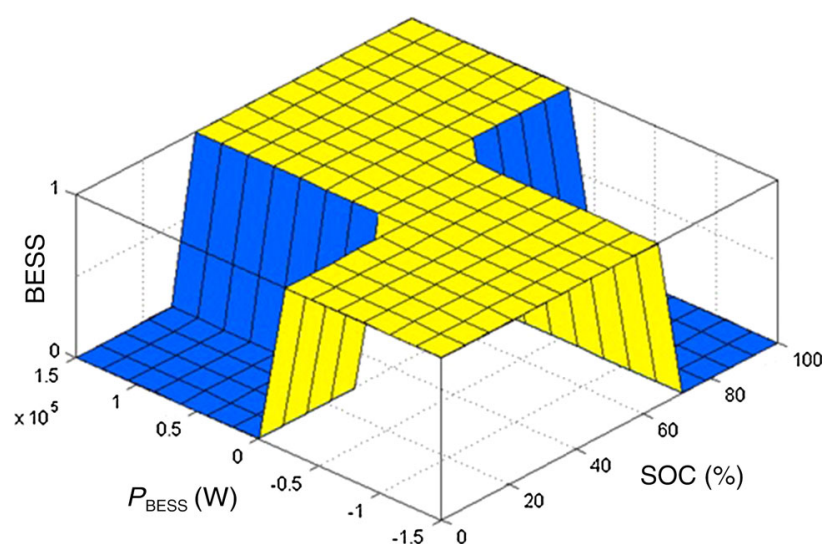

Fig. 3 Global controller control surface, BESS status at varying $P_{\mathrm{BESS}}$ and SOC

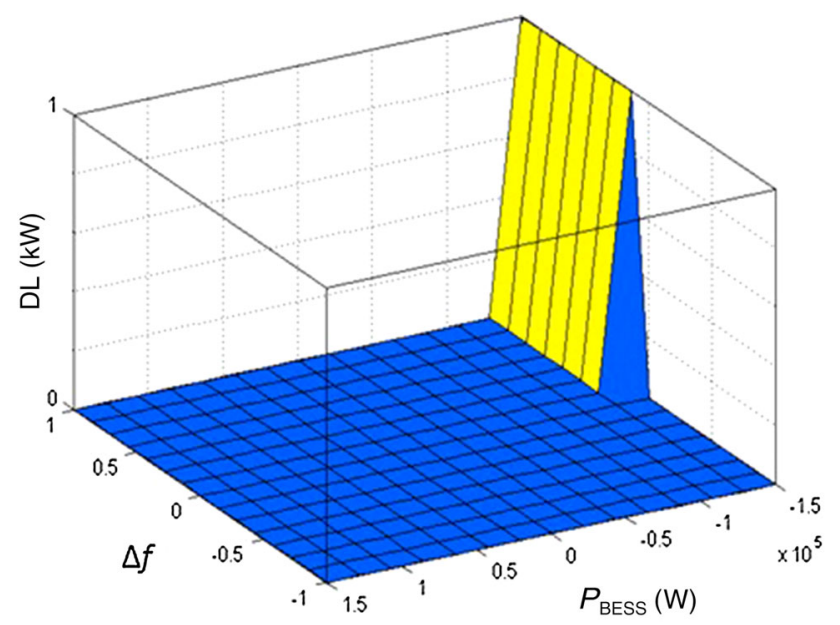

Fig. 4 Global controller, DL status at varying $\Delta f$ and $P_{\text {BESS }}$

\subsection{DL frequency control}

Fuzzy frequency controllers have been proposed in the literature. In [1], a fuzzy logic control was designed to modify the DL consumption according to the system $\Delta f$ and its rate of change $(\mathrm{d} \Delta f / \mathrm{d} t)$.

The fuzzy frequency control designed in this paper follows the same structure as the control in [1]; however, the membership functions designed here present different widths. The controller design is based on the results of a previously used PID controller. The PID controller was recreated from study [7].

The output membership functions of the proposed fuzzy frequency controller are shown in Fig. 5. The control surface is depicted in Fig. 6. It can be seen from Fig. 5 that the large membership functions are wider and output large $\Delta P$ variations, thus providing rapid control action. In contrast, the narrow shaped $\Delta P$ membership functions prevent oscillations around the settling point.

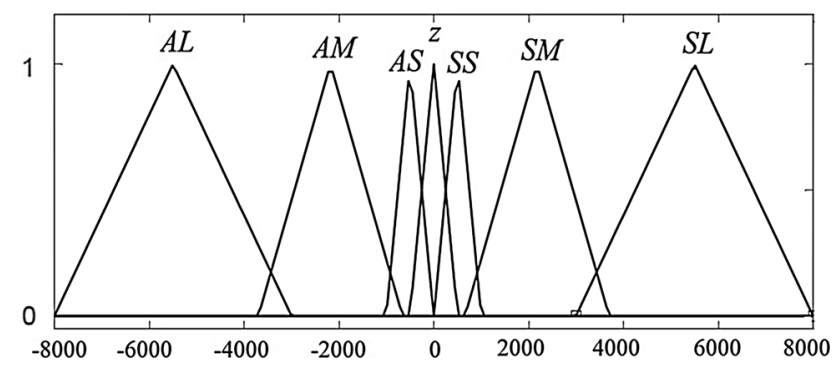

Fig. 5 DL frequency controller output membership functions

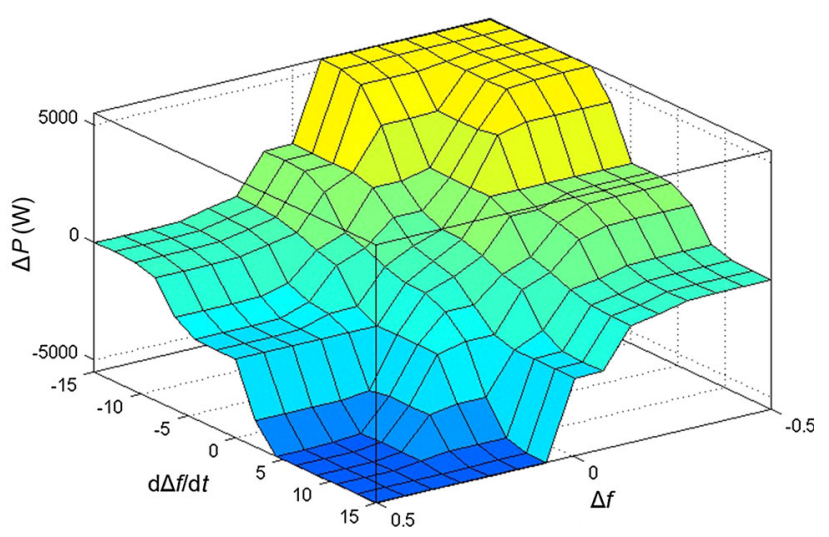

Fig. 6 DL frequency controller control surface

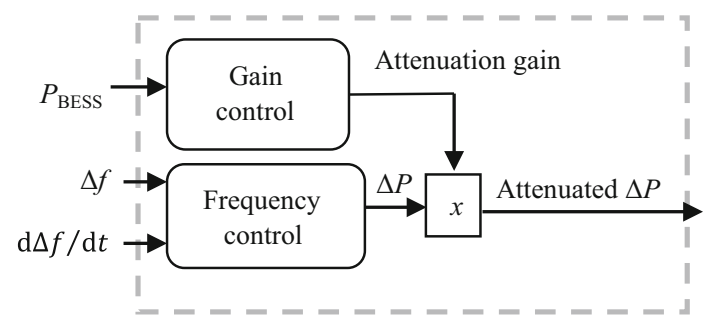

Fig. 7 Graphic representation of BESS frequency control

\subsection{BESS frequency control}

The BESS and DL share the same fuzzy inference engine for frequency control. Hence, the BESS power reference also uses the control surface in Fig. 6. However, the BESS frequency controller has been modified by introducing a controlled attenuation. The attenuation prevents undershoots and overshoots when $P_{\mathrm{BESS}}$ is close to instantaneous power limits, thereby preventing unnecessarily reaching the BESS operational limits and unnecessarily committing the secondary balancing mechanisms.

The attenuation is provided by a gain control. The gain control operates according to $P_{\mathrm{BESS}}$ and uses fuzzy logic to calculate the required attenuation. A graphic representation of the BESS frequency control is given in Fig. 7. 


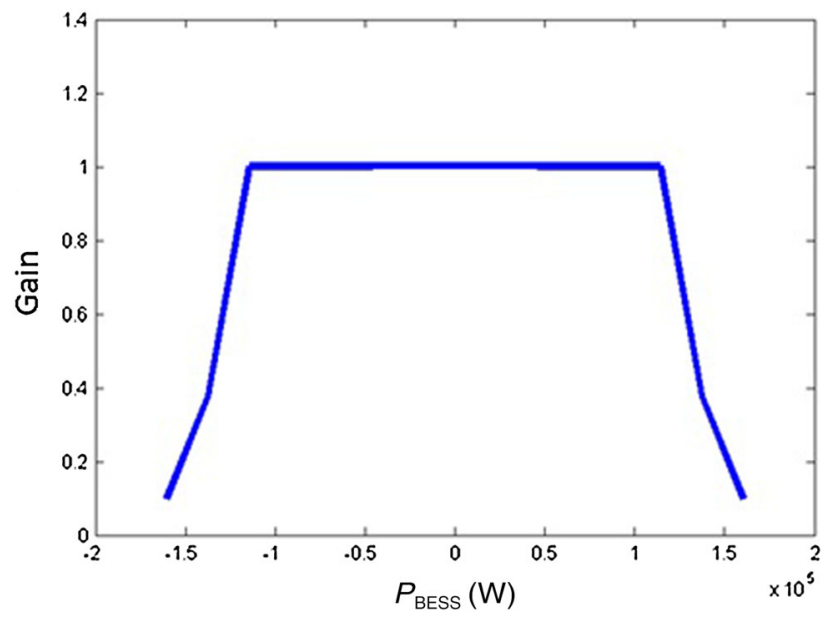

Fig. 8 Gain control, control surface

The gain control, control surface, is depicted in Fig. 8. The control surface was designed by testing different attenuation levels for a given scenario. The lowest attenuations that prevented the commissioning of the secondary balancing mechanisms were chosen. The gain control effectiveness is discussed later in Sect. 7.

The attenuated $\Delta P$ is then used to calculate the BESS power reference $\left(P_{\mathrm{BESS}}{ }^{*}\right)$ using the previous state of $P_{\mathrm{BESS}} *$. Then, a current injection control $[20,21]$ transforms $P_{\mathrm{BESS}} *$ into a voltage reference for the BESS PWM.

\subsection{BESS unload controller}

The unload controller is used to reduced $P_{\mathrm{BESS}}$ to 0 when the SOC limits are likely to be surpassed. The unload controller consists of a fuzzy inference engine that reduces the BESS absorption or consumption through $\Delta P$ variations according to the actual value of $P_{\mathrm{BESS}}$. The control surface is shown in Fig. 9.

\subsection{Engine controller}

The diesel engine provides frequency regulation through the speed governor. Thus, in this study a controller has been designed to operate the diesel engine reference speed according to the system needs. The designed engine controller anticipates the commissioning of the DG and prepares the diesel engine for commissioning by running in stand-by mode. The diesel engine runs in stand-by mode if: 1) the SOC is close to the empty limit, 2) $P_{\mathrm{BESS}}$ is close to the instantaneous supply limit.

\subsubsection{SOC close to the empty limit}

When the SOC is close to being empty, the diesel engine is commanded to run close to nominal speed. It was

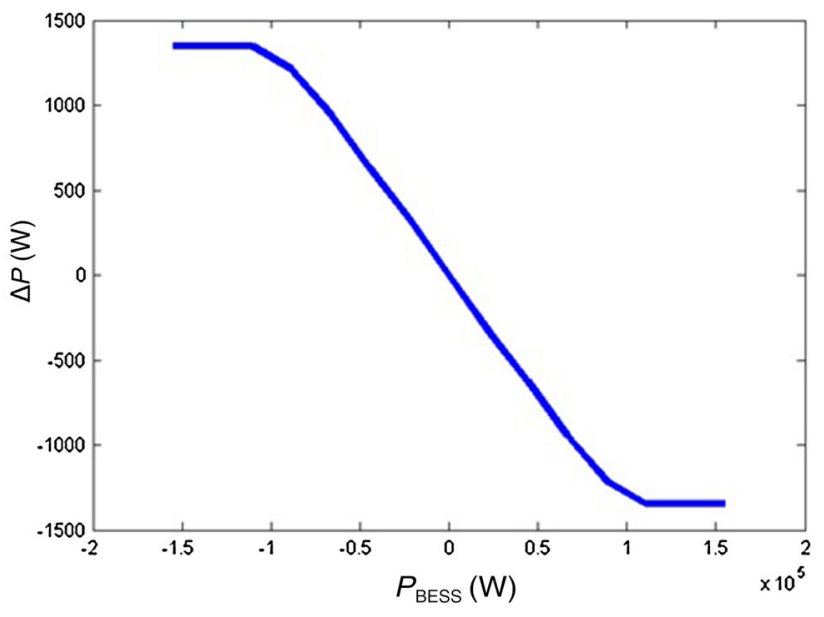

Fig. 9 BESS unload controller, control surface

concluded through simulations that the studied diesel engine requires $3.1 \mathrm{~s}$ to reach operational speed. Thus, the diesel engine must start its preparation $3.1 \mathrm{~s}$ before the SOC is empty.

To calculate the time left before the SOC is empty, the Peukert time capacity, $T_{\mathrm{p}}$ (hour), is calculated as in (1) [26]; then, the discharge speed $v_{\mathrm{d}}(\% / \mathrm{s})$ is calculated using (2).

$T_{\mathrm{p}}=\frac{R_{\text {rating }}}{\left(\frac{i_{\text {batt }} R_{\text {rating }}}{Q}\right)^{n}}$

$v_{\mathrm{d}}=\frac{100 \%}{T_{\mathrm{p}} 3600}$

where $Q$ is the battery capacity; $R_{\text {rating }}$ is the time rating of $Q ; n$ is the battery Peukert coefficient, and $i_{\text {batt }}$ is the battery current.

Using the discharge speed from (2), the time left before the SOC is empty, $t_{\mathrm{SOC}}$, is calculated through (3). The diesel engine reference speed, $\omega_{\mathrm{d}}{ }^{*}$, is modified according to (4). As stated in Sect. 2, the diesel engine rest state is simulated at a 0.3 p.u. speed. A stand-by speed of 0.97 p.u. has been chosen to anticipate the commissioning of the DG under this scenario.

$t_{\mathrm{SOC}}(s)=\frac{(\text { SOC }- \text { empty limit })}{\text { discharge speed }}$

$\left\{\begin{array}{cc}t_{\mathrm{SOC}} \text { is less } 3 \mathrm{~s}\left(t_{\mathrm{SOC}} \leq 3.1\right) & \omega_{\mathrm{d}} *=0.97 \text { p.u. } \\ t_{\mathrm{SOC}} \text { is more } 3 \mathrm{~s}\left(t_{\mathrm{SOC}}>3.1\right) & \omega_{\mathrm{d}} *=0.30 \text { p.u. }\end{array}\right.$

\subsection{2 $P_{B E S S}$ close to the instantaneous supply limit}

Since it is not possible to estimate when the maximum instantaneous supply will be met, a fuzzy approach is used to modify $\omega_{\mathrm{d}} *$ according to $P_{\mathrm{BESS}}$. As $P_{\mathrm{BESS}}$ gets closer to the instantaneous supply limit, it is more probable that a 


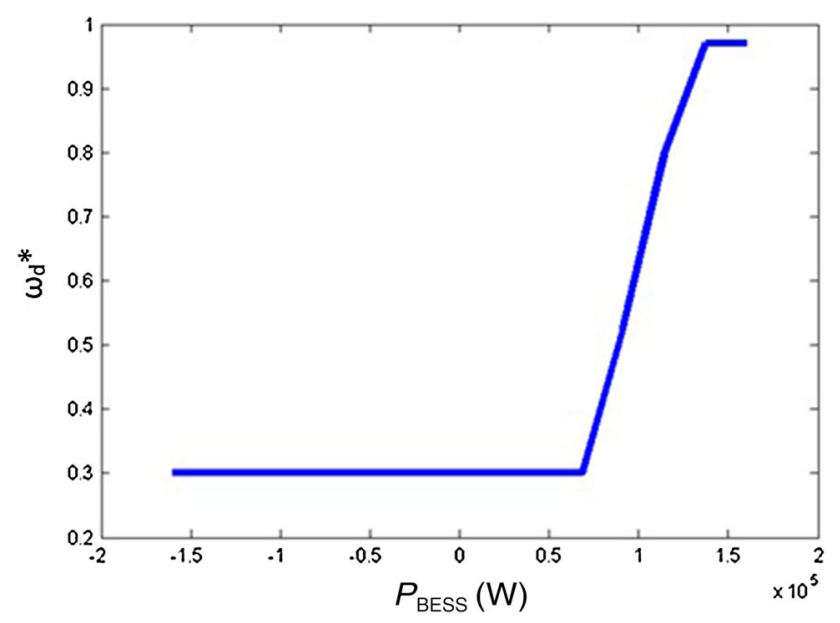

Fig. 10 Engine controller, control surface for the anticipation of the instantaneous supply limit

variation in the wind production or load consumption will drive $P_{\mathrm{BESS}}$ to the $+150 \mathrm{~kW}$ limit. Therefore, as $P_{\mathrm{BESS}}$ gets closer to the instantaneous supply limit, the diesel engine is run closer to the operational speed. The engine controller control surface for this scenario is shown in Fig. 10.

A final consideration is made for the control of the diesel engine speed. In order for the clutch to be locked, it is mandatory that the diesel engine and synchronous machine speed be almost equal. Hence, the engine control is prepared for the scenario where the diesel engine speed $\left(\omega_{\mathrm{d}}\right)$ may be largely greater than synchronous machine speed $\left(\omega_{\mathrm{s}}\right)$. If this scenario were to happen, $\omega_{\mathrm{d}} *$ will be set at the curent $\omega_{\mathrm{s}}$ through a switch. A control signal named $\omega_{\text {syn }}$ has been created for this purpose and follows (5).

$\left\{\begin{array}{c}\omega_{\mathrm{d}}>\omega_{\mathrm{s}}=\omega_{\text {syn }} \text { is } \mathrm{ON}(1) \quad \omega_{\mathrm{d}} *=\omega_{\mathrm{s}} \\ \omega_{\mathrm{d}}<\omega_{\mathrm{s}}=\omega_{\text {syn }} \text { is } \operatorname{OFF}(0) \quad \omega_{\mathrm{d}} *=\omega_{\text {fuzzy }}\end{array}\right.$

A graphic representation of the engine controller is presented in Fig. 11. The final output of the engine controller is $\omega_{\mathrm{d}}{ }^{*}$, which is then used as the speed reference for the diesel engine governor. When the engine controller outputs $\omega_{\text {syn }}=1$, the governor's reference speed is equal to $\omega_{\mathrm{s}}$. When the engine

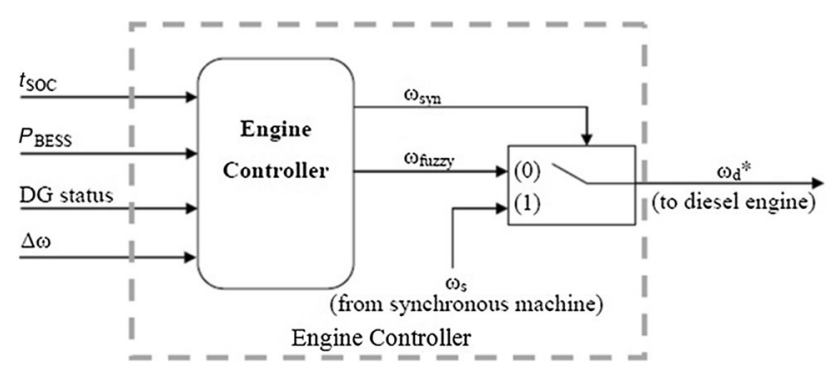

Fig. 11 Engine controller graphic representation controller outputs $\omega_{\text {syn }}=0$, the governor's reference speed is calculated by the engine controller.

\section{Simulation studies}

The proposed control strategy has been tested in Simulink. The first two scenarios test the control response to the BESS operational limits. Then, the BESS gain controller is discussed.

\subsection{Global control at SOC limits}

During initial conditions, the BESS provides frequency regulation by supplying power to the system. However, the BESS SOC approaches the empty limit and the DG must replace the BESS as frequency controller. The global controller actuation and the system response are shown in Fig. 12.

It is seen in Fig. $12 \mathrm{~b}$ that $t_{\mathrm{SOC}}$ reaches the $3.1 \mathrm{~s} \mathrm{limit}$ at $t=4.33 \mathrm{~s}$. As this occurs, the engine controller modifies $\omega_{\mathrm{d}} *$ to stand-by operation. Due to the engine's cranking delay [6], it is at $t=4.86 \mathrm{~s}$ that the diesel engine speed starts to increase, as seen in Fig. 12d. It should also be noticed from Fig. 12d that when the engine controller is not used, the engine speed is not increased until the SOC empty limit has been reached at $t=7.44 \mathrm{~s}$.

Once the SOC empty limit has been reached, the DG replaces the BESS as frequency regulator (Figs. $12 \mathrm{~g}$ and e). Simultaneously, the unload controller is committed to reduce the BESS supply to $0 \mathrm{~kW}$ (Fig. 12f).

When the engine control is used, the proposed control strategy maintains the frequency close to the reference, $60 \mathrm{~Hz}$, with a maximum $\Delta f$ of $-0.23 \%$. It is seen in Fig. $12 \mathrm{~h}$ that when the engine controller is used, the maximum $\Delta f$ can be reduced by $2.07 \%$.

\subsection{Global control at the instantaneous power absorption limit}

Due to a large power surplus, the DL starts this scenario providing frequency regulation. When a load disturbance occurs, the power surplus decreases and the BESS can alone provide frequency regulation. Simulation results are shown in Fig. 13.

During initial conditions, the BESS is absorbing at the instantaneous power limit and the DL is providing frequency regulation by absorbing the remaining power surplus (Fig. 13a). At $t=5 \mathrm{~s}$, the load consumption increases and thus the power surplus reduces.

As a result, the DL consumption reaches $0 \mathrm{~kW}$ at $t=5.05 \mathrm{~s}$. As shown in Figs. $13 \mathrm{~b}$ and $\mathrm{c}$, the global controller decommissions the DL and commits the BESS to 


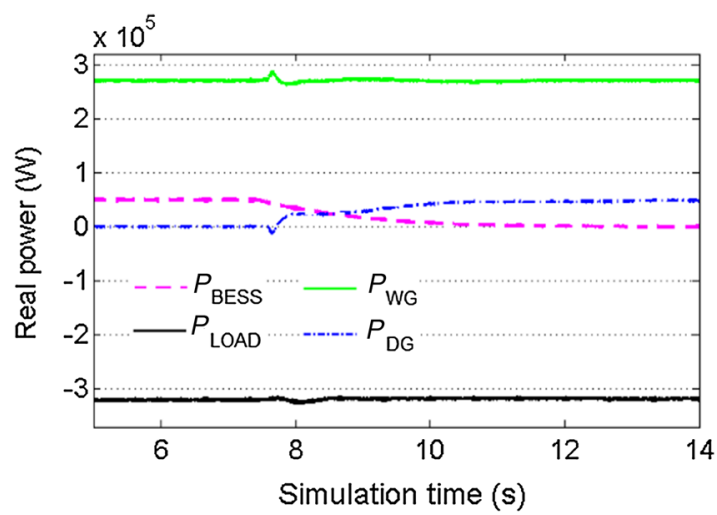

(a) Power management

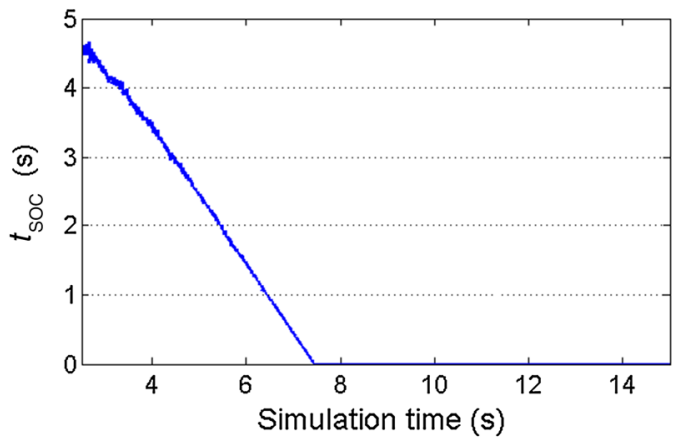

(b) $t_{\mathrm{soc}}$

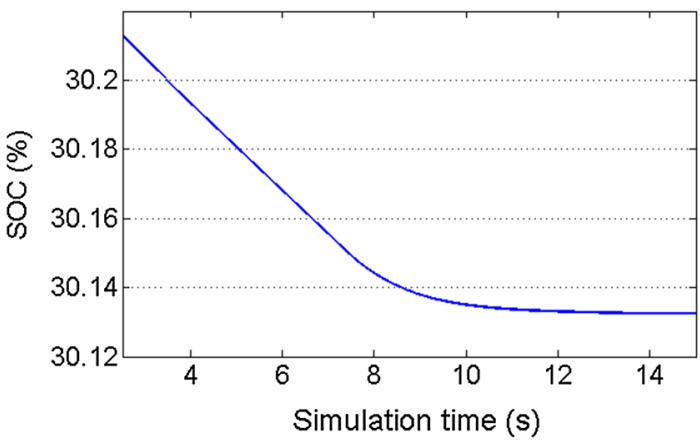

(c) $\mathrm{SOC}$

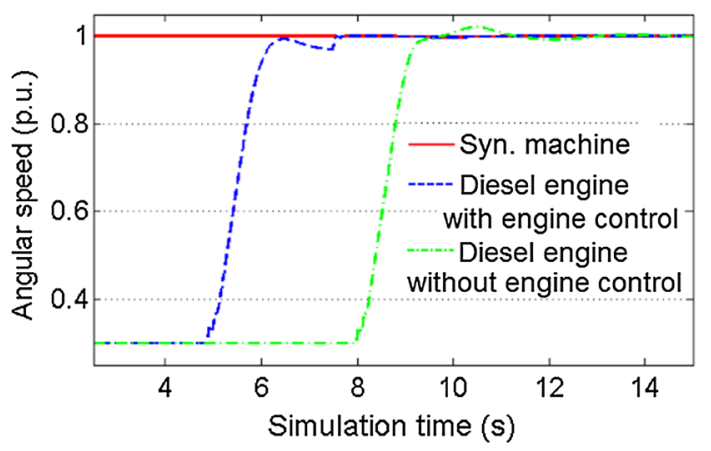

(d) DG speeds

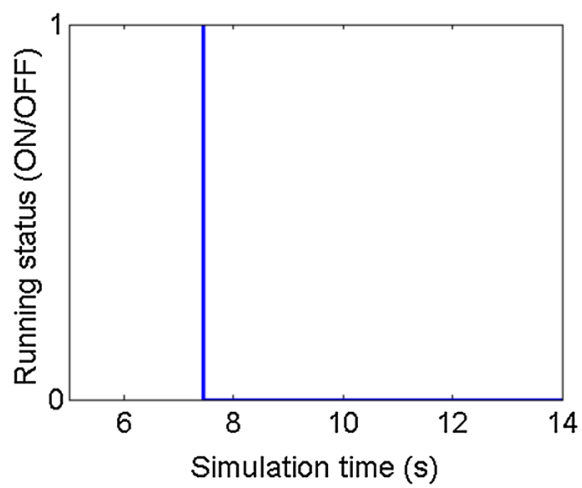

(e) BESS running status

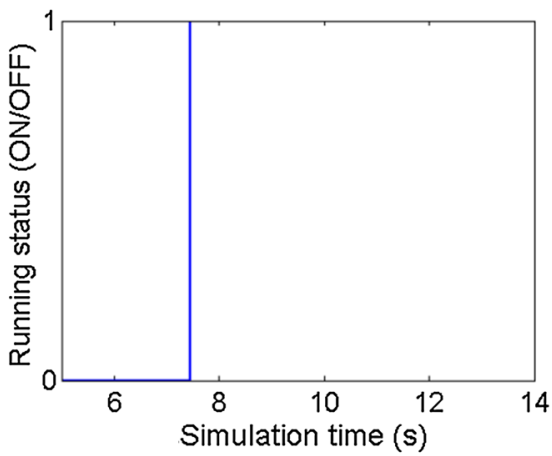

(f) Unload controller running status

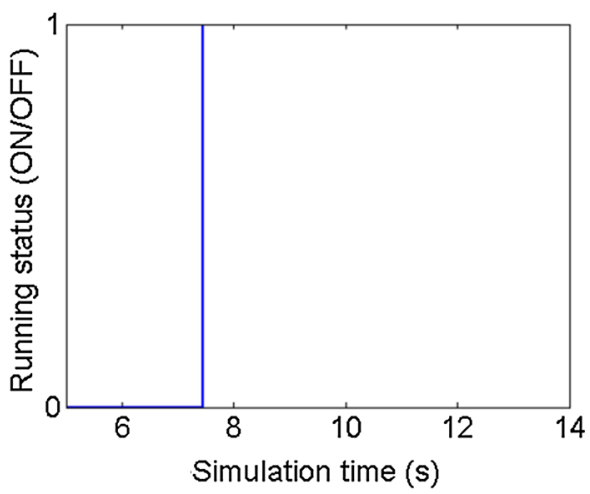

(g) DG running status

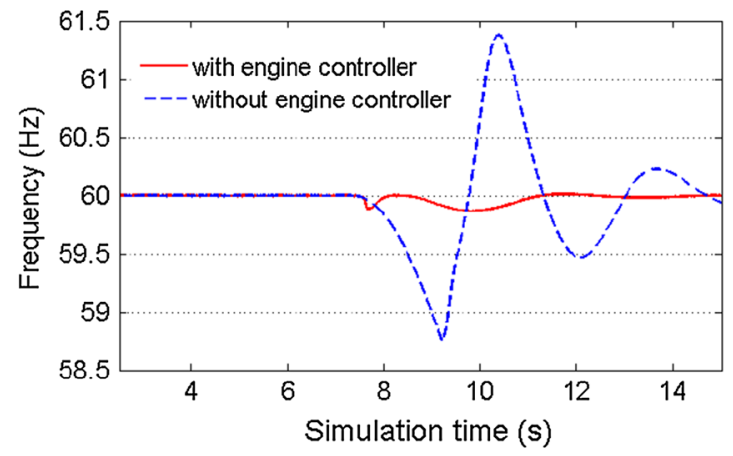

(h) Frequency

Fig. 12 Simulation results at SOC limits 


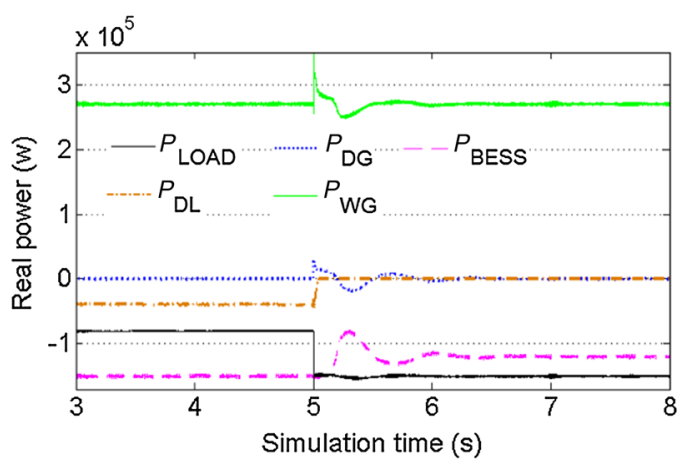

(a) Power management

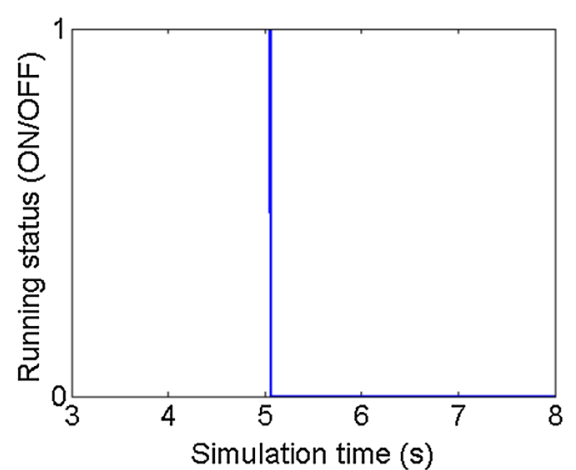

(b) DL running status

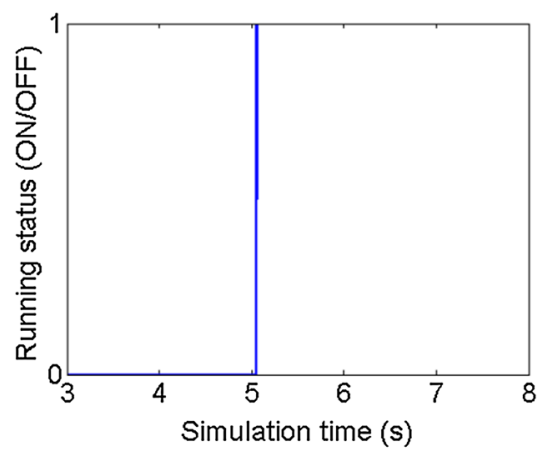

(c) BESS status

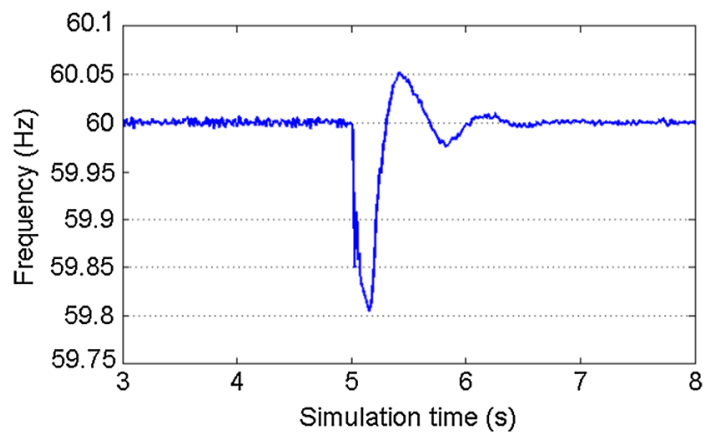

(d) Frequency

Fig. 13 Simulation results at the instantaneous power absorption limit

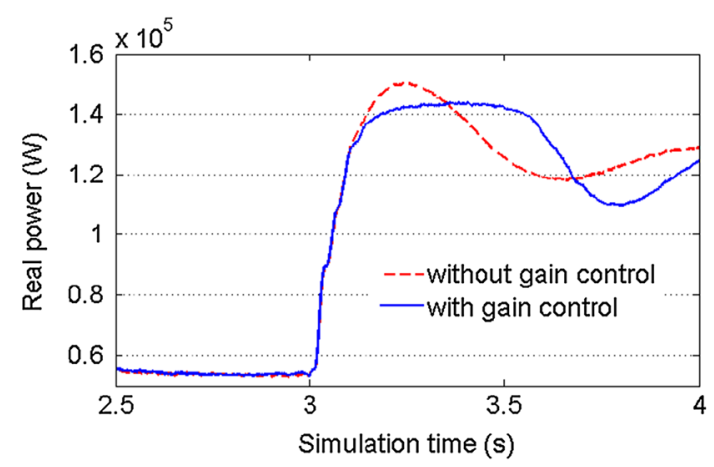

(a) BESS power output

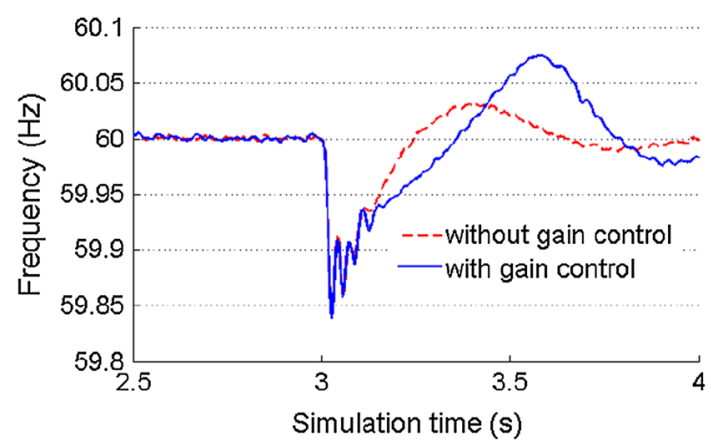

(b) Frequency

Fig. 14 Gain control performance

frequency regulation. The system frequency during the load disturbance can be observed in Fig. 13d. The maximum $\Delta f$ is $-0.32 \%$.

\subsection{BESS frequency controller}

In this scenario, the BESS consumption increases from 50.9 to $125.9 \mathrm{~kW}$. The BESS performance with and without the gain control can be seen in Fig. 14.

It is seen from Fig. 14b that the maximum $\Delta f$ is equal with and without gain control. However, due to slower frequency control, the overshoot is enlarged when the gain control is used. Simulation results show that the gain control effectively prevents the BESS power output from reaching the instantaneous supply limit; thus, preventing the unnecessary commissioning of the DG and improving the system power management.

\section{Conclusions}

A control strategy for automatic frequency regulation in an islanded microgrid is proposed. The proposed strategy 
does not require the wind power or load consumption measurements.

Wind turbines are usually located away from loads. In order to calculate the system's power mismatch, wind power and load consumption measurements may be required to be transmitted over a long distance that can increase the risk of failure on the transmission channel, causing the disruption of the power system operation. By avoiding the power mismatch calculation, the proposed control strategy can reduce the risk of operation disruption due to transmission failure.

In a power mismatch coordination approach, new measurement equipment is required as new loads or renewable sources are added to the system. As a result, the coordination approach proposed in the literature will require additional cost as the system increases in size, or as distributed loads appear across the system. In contrast, the control strategy proposed here will not require additional measurement equipment as new renewable sources or loads are introduced to the system. Hence, the proposed strategy can facilitate the introduction of other renewable energy sources.

Simulation studies show that the proposed control strategy efficiently operates the system according to the dispatch and operational guidelines. Hence, the automatic operation of the islanded microgrid is achieved. Using high-order mathematical models it is shown that the frequency is efficiently regulated with a maximum deviation observed of $0.32 \%$.

Individual controllers are also designed to operate the balancing mechanisms. The proposed engine controller greatly improves the system frequency by anticipating the SOC empty limit and preparing the DG for commissioning. A reduction of $2.7 \%$ on the frequency deviation is observed when the proposed engine controller is used. Ultimately, the proposed gain controller improves the system performance by preventing the unnecessary commissioning of secondary balancing mechanisms.

Open Access This article is distributed under the terms of the Creative Commons Attribution License which permits any use, distribution, and reproduction in any medium, provided the original author(s) and the source are credited.

\section{References}

[1] Hasanien HM, Muyeen SM, Tamura J (2009) Frequency control of isolated network with wind and diesel generators by using fuzzy logic controller. In: Proceedings of the 2009 international conference on electrical machines and systems (ICEMS'09), Tokyo,15-18 Nov 2009, 6 pp

[2] Marzband M, Sumper A, Gomis-Bellmunt O, et al (2011) Frequency control of isolated wind and diesel hybrid MicroGrid power system by using fuzzy logic controllers and PID controllers. In: Proceedings of the 11th international conference on electrical power quality and utilisation (EPQU'11), Lisbon, 17-19 Oct 2011, 6 pp

[3] Sharma S, Singh B (2010) Fuzzy proportional-integral regulators for stand-alone wind energy conversion system. In: Proceedings of the 2010 international conference on power, control and embedded systems (ICPCES'10), Allahabad, 29 Nov-1 Dec 2010, 8 pp

[4] Ko HS, Niimura T, Jatskevich J, et al (2004) Power quality control of hybrid wind power generation with battery storage using fuzzy-LQR controller. In: Proceedings of the 2004 IEEE Power Engineering Society general meeting (PES'04), Vol 2, Denver, COUSA, 6-10 Jun 2004, pp 1721-1727

[5] Yasin A, Napoli G, Ferraro M, et al (2011) Fuzzy logic based management of a stand-alone hybrid generator. In: Proceedings of the 2011 international conference on clean electrical power (ICCEP'11), Ischia, 14-16 Jun 2011, pp 690-696

[6] Sebastián R (2008) Smooth transition from wind only to wind diesel mode in an autonomous wind diesel system with a battery-based energy storage system. Renew Energ 33(3):454-466

[7] Sebastian R (2011) Modelling and simulation of a high penetration wind diesel system with battery energy storage. Int J Electr Power Energ Syst 33(3):767-774

[8] Mendis N, Muttaqi KM, Perera S, et al (2011) A novel control strategy for stand-alone operation of a wind dominated RAPS system. In: Proceedings of the Industry Applications Society annual meeting (IAS'11), Orlando, 9-13 Oct 2011, 8 pp

[9] Mendis N, Muttaqi KM, Perera S (2012) Active power management of a super capacitor-battery hybrid energy storage system for standalone operation of DFIG based wind turbines. In: Proceedings of the Industry Applications Society annual meeting (IAS'12), Las Vegas, 7-11 Oct 2012, 8 pp

[10] Mendis N, Muttaqi KM, Sayeef S, et al (2010) Autonomous operation of wind-battery hybrid power system with maximum power extraction capability. In: Proceedings of the 2010 international conference on power system technology (POWERCON'10), Hangzhou, 24-28 Oct 2010, 7 pp

[11] Hannett LN, de Mlello FP, Tylinski GH et al (1982) Validation of nuclear plant auxiliary power supply by test. IEEE Trans Power App Syst 101(9):3068-3074

[12] Yeager KE, Willis JR (1993) Modeling of emergency diesel generators in an 800 megawatt nuclear power plant. IEEE Trans Energ Conver 8(3):433-441

[13] Krause PC, Wasynczuk O, Sudhoff SD (1995) Analysis of electric machinery. Wiley, New York

[14] Heier S (2006) Grid integration of wind energy conversion systems. Wiley, New York

[15] Pao LY, Johnson KE (2011) Control of wind turbines. IEEE Contr Syst 31(2):44-62

[16] Tremblay O, Dessaint LA, Dekkiche AT (2007) A generic battery model for the dynamic simulation of hybrid electric vehicles. In: Proceedings of the 2007 IEEE vehicle power and propulsion conference (VPPC'07), Arlington, 9-12 Sept 2007, pp 284-289

[17] Garimella N, Nair NC (2009) Assessment of battery energy storage systems for small-scale renewable energy integration. In: Proceedings of the 2009 IEEE Region 10 conference (TENCON'09, 23-26 Jan 2009, 6 pp

[18] Mulder G, Omar N, Pauwels S et al (2013) Comparison of commercial battery cells in relation to material properties. Electrochim Acta 87(1):473-488

[19] Lee J, Kim Y, Cha H (2011) A new battery parameter identification considering current, SOC and Peukert's effect for hybrid electric vehicles. In: Proceedings of the 2011 IEEE energy conversion congress and exposition (ECCE'11), Phoenix, 17-22 Sept 2011, pp 1489-1494 
[20] Mendis N, Muttaqi KM, Sayeef S et al (2010) Application of a hybrid energy storage in a remote area power supply system. In: Proceedings of the 2010 IEEE international energy conference and exhibition (EnergyCon'10), Manama, 18-22 Dec 2010, pp 576-581

[21] Sebastián R, Peña Alzola R (2011) Simulation of an isolated wind diesel system with battery energy storage. Electr Power Syst Res 81(2):677-686

[22] Ko HS, Yoon GG, Hong WP, et al (2007) Control of hybrid wind power generation system with dump load using advanced fuzzy-robust controller. In: Proceedings of the international conference on electrical machines and systems (ICEMS'07), Seoul, 8-11 Oct 2007, pp 219-226

[23] IEEE Std 421.5-1992 IEEE recommended practice for excitation system models for power system stability studies. 1992

[24] Zhu WH, Zhu Y, Davis Z et al (2013) Energy efficiency and capacity retention of $\mathrm{Ni}-\mathrm{MH}$ batteries for storage applications. Appl Energ 106:307-313

[25] Wood AJ, Wollenberg BF, Sheble GB (1996) Control of generation. Power generation operation and control, 2nd edn. Wiley, New York, pp 336-340

[26] Hausmann A, Depcik C (2013) Expanding the peukert equation for battery capacity modeling through inclusion of a temperature dependency. J Power Sources 235:148-158
Pamela MANJARRES obtained her B.Sc. degree from the Universidad Rafael Urdaneta (URU) in Maracaibo, Venezuela, in 2011. Later she enrolled in the University of Calgary where she successfully completed her M.Sc. studies under the supervision of Dr. Om Malik. Based on her interest in renewable energy, Pamela's research focused on the automation of hybrid power systems. Pamela is currently working in the industry.

Om MALIK obtained a Master's Degree from Roorkee University, India in 1962, a Ph.D. from London University and a DIC from the Imperial College, London in 1965. He was teaching and researching in Canada from 1966 to 1997. Today he continues researching as Professor emeritus at the University of Calgary, Canada. He was President of IEEE Canada and is currently President of the Engineering Institute of Canada. Professor MALIK is a Life fellow of IEEE, and a Fellow of the Engineering Institute of Canada, Canadian Academy of Engineering, Institution of Engineering and Technology, Engineers Canada and World Innovation Foundation. He has done pioneering work in the development of adaptive and artificial intelligence based controllers for electric power systems, and digital and artificial intelligence based protection schemes. Publications include over 700 papers. 